\title{
EFFETS DE LA DÉCURARISATION POST-OPERATOIRE PAR LA NÉOSTIGMINE SUR LES ANASTOMOSES DIGESTIVES
}

\author{
P. Morisot; ${ }^{\circ}$ J. Loygue, $\nmid$ et Cl. Gullmet
}

LA SUPPRESSION des effets résiduels des curares curarimimétiques par la néostigmine à la fin de l’anesthésie est un fait bien établi et largement utilisé. Son principale avantage est certainement de restituér aux malades une bonne ventilation. ${ }^{3,6-9,12,14}$

Malheureusement la néostigmine n'est pas dépourvue d'effets secondaires indésirables. Les plus connus et les plus redoutés sont ses effets cardio-vasculaires. Mais en 1968 Bell et Lewis ${ }^{2}$ en signalaient un autre tout aussi redoutable : la désunion des anastomoses intestinales par l'hyperpéristaltisme qu'elle produit. D'après leur propre expérience, le taux de fistulisation des anastomoses iléo-rectales après colectomie totale pour colite ulcéreuse était accru de façon significative ( 36 pour cent contre 4 pour cent) selon qu'ils avaient ou non utilisé la néostigmine à la fin de l'intervention.

Ce travail se propose de vérifier si « beaucoup de fistules intestinales post-opératoires peuvent être imputées à la néostigmine » comme le suggèrent ces auteurs.

\section{Materiel et Methode}

Cette étude prospective a porté sur 400 opérés régulièrement inscrits au programme opératoire du Service de Chirurgie Digestive (Pr Loygue) de l'Hôpital St Antoine à Paris, pendant une période d'un an. Leur âge et leur catégorie selon la classification de la Société des Anesthésiologistes Américains sont indiqués dans le Tableau I, l'intervention qu'ils ont subie dans le Tableau II. Ils ont été répartis par tirage au sort en deux groupes de 200 malades chacun : 200 décurarisés par la néostigmine, 200 qui ne l'ont pas été.

Le dépistage des fistules anastomotiques post-opératoires a été laissé à l'appréciation de chaque chirurgien qui a toujours ignoré à quel groupe appartenaient ses malades. Et sur le seul critère d'un examen clinique quotidien les malades ont été classés en quatre séries :

1. fistulc certaine : lorsque du liquide digestif s'est fait jour par le système de draînage chirurgical et/ou devant les signes caractéristiques d'une péritonite localisée ou généralisée ;

2. absence de fistule : lorsque rien ne pouvait la faire suspecter ;

-Département d'Anesthésiologie, Hôpital Saint Antoine, Paris. L'adresses actuelle du Docteur Morisot est au Département D'Anesthésie-Réanimation, Hôpital Intercommunal, 91160 Longjumeau.

†Professeur, Département de Chirurgie Digestive, Hôpital Saint-Antoine, Paris.

†Professeur Agrégé, Département d'Anesthésiologie, Hôpital Saint Antoine, 184 rue du Faubourg St Antoine, 75571 Paris 12. 
MORISOT, et al.: EFFETS DE LA DÉCURARISATION POST-OPÉRATOIRE

TABLEAU I

REPARTITION DES MALADES SELON L'ÂGE ET LA CATÉGORIE (A.S.A.)

\begin{tabular}{lrrrrrr}
\hline \hline & \multicolumn{7}{c}{ Catégorie } & \\
\cline { 2 - 6 } $\begin{array}{c}\text { Age } \\
\text { années) }\end{array}$ & \multicolumn{1}{c}{$\mathbf{1}$} & 2 & 3 & $\mathbf{4}$ & 5 & Total \\
\hline $10-19$ & 5 & 2 & - & - & - & $7-1,75 \%$ \\
$20-29$ & 20 & 5 & 1 & - & - & $26-6,50 \%$ \\
$30-39$ & 27 & 1 & 1 & - & - & $29-7,25 \%$ \\
$40-49$ & 38 & 13 & 2 & - & - & $53-13,25 \%$ \\
$50-59$ & 39 & 26 & 1 & - & - & $66-16,50 \%$ \\
$60-69$ & 60 & 44 & 11 & - & - & $115-28,75 \%$ \\
$70-79$ & 35 & 34 & 7 & - & - & $76-19 \%$ \\
$80-89$ & 11 & 15 & 1 & 1 & - & $28-11 \%$ \\
Total & 235 & 140 & 24 & 1 & - & $400-100 \%$ \\
\hline
\end{tabular}

TABLEAU II

REPARTITION DES MALADES SELON L'INTERVENTION

\begin{tabular}{lccr}
\hline & \multicolumn{2}{c}{ Néostigmine } & \\
\cline { 2 - 3 } & sans & avec & Total \\
\hline Oesophage, Estomac & 53 & 48 & 101 \\
Voies biliaires, Foie, & & & \\
Pancréas, Duodénum & 38 & 49 & 87 \\
Jéjunum, Iléon & 25 & 19 & 44 \\
Colon & 83 & 82 & 165 \\
Autres & 1 & 2 & 3 \\
$\quad$ Total & 200 & 200 & 400 \\
\hline
\end{tabular}

3. fistule probable et

4. fistule improbable lorsqu'un incident clinique (trouble du transit, foyer infectieux abdominal, fièvre inexpliquée) laissait craindre la possibilité d'une fistule, sans qu'aucun argument absolu ne permettre de l'affirmer mi de la rejeter.

Notre protocole anesthésique a été le même pour tous les malades. Après prémédication le plus souvent par le mélange prométhazine-péthidine-atropine, l'induction a été faite par injection intraveineuse de penthiobarbital ( $331 \mathrm{mg} \pm 87)^{\circ}$ et succinylcholine ( $48 \mathrm{mg} \pm 7$ ), puis les malades ont été intubés, ventilés mécaniquement et l'anesthésie entretenue par le protoxyde d'azote $(50$, p. 100$)$ et la phénopéridine (de $2 \mathrm{mg} \pm 0,6$ pour les interventions d'une heure ou moins, à 5,1 $\mathrm{mg} \pm 1,7$ pour les interventions de six heures ou davantage), à l'exclusion de tout autre analgésique. Une cent vingt-trois malades ont reçu en plus du diazepam à dose modérée $(10,3 \mathrm{mg} \pm 5,0)$. Le relâchement musculaire a été obtenu par la d-tubocurarine, à l'exclusion de tout autre curarisant, et toujours sous le double contrôle de la clinique et d'un stimulateur de nerf périphérique de ChurchillDavidson. La dose moyenne de d-tubocurarine utilisée a été de $56,3 \mathrm{mg} \pm 16$ pour une durée moyenne d'intervention de $3 \mathrm{~h} 39 \mathrm{mn} \pm 1 \mathrm{~h} 26 \mathrm{mn}$.

`Les chiffres indiqués sont : la moyenne \pm l'écart-type. 
TABLEAU III

FRÉQUENCE COMPARÉE DES FISTULES POST-OPł́RATOIRES DANS LES DEUX SÉRIES DE MALADES

\begin{tabular}{lrrr}
\hline & \multicolumn{2}{c}{ Néostigmine } & \\
\cline { 2 - 3 } & sans & avec & Total \\
\hline 1. Fistule certaine & 16 & 18 & 34 \\
2. Absence de fistule & 180 & 173 & 353 \\
3. Fistule probable & 1 & 1 & 2 \\
4. Fistule improbable & 3 & 8 & 11 \\
$\quad$ Total & 200 & 200 & 400 \\
\hline
\end{tabular}

TABLEAU IV

NATURE DES ANASTOMOSES QUI ONT FISTULISE

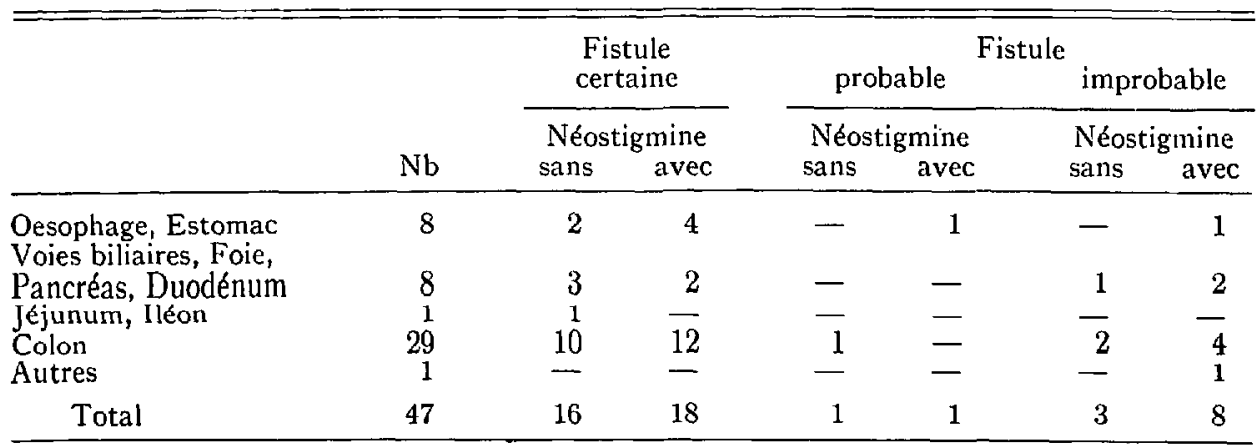

A la fin de l'intervention les 200 malades non décurarisés ont été conduits en salle de réveil et ventilés jusqu'au retour d'une ventilation spontanée parfaite. Les 200 malades désignés pour être décurarisés ont reçu une injection intraveineuse du mélange atropine-prostigmine à la dose de $1 \mathrm{mg}$ d'atropine et $2,5 \mathrm{mg}$ de néostigmine pour 187 d'entre eux, à une dose moindre pour sept autres, et à une dose supérieure (maximum $3,5 \mathrm{mg}$ de néostigmine) pour les six malades restants.

\section{Resultats}

Aucune différence significative n'apparaît entre les deux groupes de malades en ce qui concerne l'âge, le sexe, l'état général (indice A.S.A.), l'intervention et le nombre d'anastomoses.

La fréquence des désunions anastomotiques est indiquée dans le Tableau III. Le Tableau IV précise la nature des anastomoses qui ont fistulisé. Il n'existe pas de différence significative dans la survenue des fistules anastomotiques entre les deux groupes de malades : ceux qui ont reçu de la néostigmine et ceux qui n'en ont pas reçue $\left(\chi^{2}=0,122, p<0,01\right)$. Si on ne tient compte que des résultats certains en excluant les deux séries de résultats " probables * et "improbables ", la différence entre les deux groupes n'est pas davantage significative $\left(\chi^{2}=0,185, \mathrm{p}<0,01\right)$.

En outre les suites opératoires des deux groupes de malades sont tout à fait semblables (Tableau V). 
TABLEAU $\mathrm{V}$

COMPaRAISON DES SUITES OPÉratoIRES DANS LES DEUX SÉRIES DE MALADES

\begin{tabular}{|c|c|c|c|}
\hline & \multicolumn{2}{|c|}{ Néostigmine } & \multirow[b]{2}{*}{$p$} \\
\hline & sans & avec & \\
\hline $\begin{array}{l}\text { Premier gaz (jours) } \\
\text { Première selle (jours) } \\
\text { Complications post- }\end{array}$ & $\begin{array}{c}3 \pm 1 \\
4,5 \pm 1,7\end{array}$ & $\begin{array}{c}3 \pm 1 \\
4,5 \pm 1,7\end{array}$ & $\begin{array}{l}\text { N.S. } \\
\text { N.S. }\end{array}$ \\
\hline $\begin{array}{l}\text { Complications post- } \\
\text { opératoires diverses } \\
\text { (pour cent) }\end{array}$ & & 14,75 & N.S. \\
\hline $\begin{array}{l}\text { Réinterventions (pour cent) } \\
\text { Décès post-opératoires }\end{array}$ & 1,50 & 1,75 & N.S. \\
\hline $\begin{array}{l}\text { (pour cent) } \\
\text { Cathétérisme vésical }\end{array}$ & 2,75 & 3 & N.S. \\
\hline $\begin{array}{l}\text { (pour cent) } \\
\text { Sonde vésicale à demeure }\end{array}$ & 2 & 2 & N.S. \\
\hline $\begin{array}{l}\text { (pour cent) } \\
\text { Durée d'hospitalisation }\end{array}$ & 5 & 8,75 & N.S. \\
\hline (jours) & $16 \pm 8,6$ & $16,8 \pm 12,8$ & N.S. \\
\hline
\end{tabular}

\section{Conclusion}

Nos résultats, en accord avec ceux de Brown et col., ${ }^{4}$ s'opposent radicalement à ceux de Bell et Lewis. ${ }^{2}$ Cette contradiction tient peut-être à deux différences entre ces deux études :

1. Le dépistage des fistules anastomotiques : uniquement radiologique dans le travail de Bell et Lewis (lavement baryté à la troisième semaine post-opératoire), il a été seulement clinique pour nos malades. Un certain nombre de petites fuites anastomotiques peuvent ne donner lieu à aucun signe clinique et donc avoir échappé à notre étude.

2. La sélection des malades : limitée par Bell et Lewis aux cas de colectomie totale avec anastomose iléo-rectale pour colite ulcéreuse, nous l'avons étendue sans distinction à tous les malades du programme opératoire d'un service de chirurgie. Or les anastomoses iléo-rectales pour colite ulcéreuse sont réputées pour leur particulière fragilité.

Quels que soient les effets pharmacologiques de la néostigmine sur la motricité intestinale, $, 1, \bar{\pi}, 7,10,11,13$ son utilisation au moins à la dose où nous l'avons employée ne nous paraît pas devoir compromettre la cicatrisation des anastomoses digestives. Un travail expérimental récent ${ }^{15}$ tend à confirmer cette inocuité.

\section{RÉSUMÉ}

Les effets de la décurarisation post-opératoire par la néostigmine sur les anastomoses digestives ont été étudiés en double insu et par tirage au sort sur une série de 400 malades. Aucune différence significative dans le taux des fistules anastomotiques n'a été trouvée entre les deux groupes de malades, l'un décurarisé par la néostigmine, l'autre non.

\section{SUMMARY}

A prospective study was undertaken to assess the influence of neostigmine, a 
reversal agent for curarimimetic myorelaxants, on the incidence of postoperative disruption of anastomotic sites. Over a period of one year, 400 patients had surgery, including anastomosis, on the digestive tract for a variety of surgical conditions (Table II). At the end of anaesthesia, 200 patients received doses of atropine and neostigmine, usually $1 \mathrm{mg}$ and $2.5 \mathrm{mg}$ of each, as indicated on clinical basis and neuromuscular stimulation. The other patients did not receive these drugs and were ventilated till the myorelaxation vanished spontaneously.

During the postoperative period the incidence of anastomotic breakdown was assessed by the surgeon, unaware of the use or the omission of neostigmine in his patients. Anastomotic leakage was classified in four groups, namely: proved, absent, likely and unlikely.

In this series and according to these clinical criteria, both groups had an incidence of anastomotic breakdown which was not significantly different (Table III). Neostigmine as used in this work does not seem to compromise the normal healing of anastomotic sites on the digestive tract.

\section{BIBLIOGRAPHIE}

1. BARANY, F. Endoradiosonde study of propulsion and pressure activity induced by test meals, prostigmine and diphenoxylate in the small intestine. Gut. 5: 90 (1970).

2. BELL, C.M.A. \& LEWIS, C.B. Effect of neostigmine on integrity of ileo-rectal anastomoses. Brit. Med. J. 3: 587 (1968).

3. Baidenbaugh, P.O. \& Chunchill-Davidson, H.C. Response to tubocurarine chloride and its reversal by neostigmine methylsulfate in man. J.A.M.A. 203: 541 (1968).

4. Brown, E.N., Dauchty, M.J., \& Clayton PeTtY, W. Integrity of intestinal anastomoses following muscle relaxant reversal with neostigmine. Anesth. Analg. Curr. Res. 52: 117 (1973).

5. Chaudary, N.A. \& Truelove, S.C. Human colonic motility: a comparative study of normal subjects, patients with ulcerative colitis, and patients with the irritable colon syndrome. 1. Resting patterns, 2. The effects of prostigmine, 3. Effects of emotion. Gastroenterology 40: $1(1961)$.

6. Doughty, A.G. \& Wylie, W.D. Antidotes to "true" curarizing agents. Brit. J. Anaesth. 24 : 66 ( 1952$)$.

7. Goodman, L.S. \& Gilman, A. The pharmacological basis of therapeutics. 4 th ed. The Macmillan Co., New York; N.Y. pp. 442-465 (1970).

8. Hannington-KIfF, J.G. Timing of atropine and neostigmine in the reversal of muscle relaxants. Brit. Med. J. 1: 418 (1969).

9. KATZ, R.L. Neuromuscular effects of d-tubocurarine, edrophonium and neostigmine in man. Anesthesiology 28: 327 (1963).

10. Painter, N.S. The intraluminal pressure patterns in diverticulosis of the colon. III. The effect of prostigmine. Gut. 5: 365 (1964).

11. Painter, N.S. The effect of neostigmine methyl sulfate on the human colon in health and diverticulosis. Proc. Roy. Soc. Med., 60: 219 (1967).

12. Riding, J.E. \& Robinson, J.S. The safety of neostigmine. Anaesthesia 16: 346 (1961).

13. Wilkins, J.S., Hardeaste, J.D., Man, C.V., \& Coll. Effects of neostigmine and atropine on motor activity of ileum, colon and rectum of anesthetized subjects. Brit. Med. J. 1: $793(1970)$.

14. Wyllie, W.D. \& Churchill-Davidson, H.C. A practice of anaesthesia, 3rd ed, LloydLuke Ltd, London, 1972, pp. 808-937.

15. Yellin, A.E., Newman, J., \& Donovon, A.J. Neostigmine-induced hyperperistalsis. Effects on security of colonic anastomoses. Arch. Surg. 106: 779 (1973). 